\title{
Integrated analysis reveals five potential ceRNA biomarkers in human lung adenocarcinoma
}

\author{
Yu Liu ${ }^{1}$, Deyao Xie ${ }^{1}$ ， Zhifeng He ${ }^{1}$ ， Liangcheng Zheng ${ }^{\text {Corresp. } 1}$ \\ ${ }^{1}$ Department of Thoracic surgery, The First Affiliated Hospital of Wenzhou Medical University, Wenzhou, Zhejiang, China \\ Corresponding Author: Liangcheng Zheng \\ Email address: zlc201810@126.com
}

Background. ceRNAs (competing endogenous RNAs) are a newly identified type of regulatory RNA. Accumulating evidence suggests that ceRNAs play an important role in the pathogenesis of diseases such as cancer. Thus, ceRNA dysregulation may represent an important molecular mechanism underlying cancer progression and poor prognosis. In this study, we aimed to identify ceRNAs that may serve as potential biomarkers for early diagnosis of lung adenocarcinoma (LUAD). Methods. We performed differential gene expression analysis on TCGA-LUAD datasets to identify differentially expressed (DE) mRNAs, IncRNAs, and miRNAs at different tumor stages. Based on the ceRNA hypothesis and considering the synergistic or feedback regulation of ceRNAs, a IncRNA-miRNA-mRNA network was constructed. Functional analysis was performed using GO term and KEGG pathway enrichment analysis and KOBAS 2.0 software. Transcription factor analysis was carried out to identify direct targets of the transcription factors associated with LUAD prognosis. Identified differentially expressed genes were validated using GEO datasets. Results. Based on analysis of TCGA- LUAD datasets, we obtained 2610 DE mRNAs, 915 IncRNAs, and 125 miRNAs that were common to different tumor stages ( $\mid \log _{2}$ (Fold change)| $\geq 1$, FDR $<0.01$ ), respectively. Functional analysis showed that the aberrantly expressed mRNAs were closely related to tumor development. Survival analyses of the constructed ceRNA network modules demonstrated that five of them exhibit prognostic significance. The five ceRNA interaction modules contained one IncRNA (FENDRR), three mRNAs (EPAS1, FOXF1, and EDNRB), and four miRNAs (hsa-miR-148a, hsa-miR-195, hsa-miR-196b, and hsa-miR-301b). The aberrant expression of one IncRNA and three mRNAs was verified in the LUAD GEO dataset. Transcription factor analysis demonstrated that EPAS1 directly targeted 13 DE mRNAs. Conclusion. Our observations indicate that IncRNA-related ceRNAs and TFs play an important role in LUAD. The present study provides novel insights into the molecular mechanisms underlying LUAD pathogenesis. Furthermore, our study facilitates the identification of potential biomarkers for the early diagnosis and prognosis of LUAD and therapeutic targets for its treatment. 


\section{Integrated analysis reveals five potential ceRNA}

\section{2 biomarkers in human lung adenocarcinoma}

3

9 Corresponding Author:

Liangcheng Zheng ${ }^{1}$

\section{Abstract} adenocarcinoma (LUAD). GEO datasets.

Yu Liu ${ }^{1}$, Deyao Xie ${ }^{1}$, Zhifeng He ${ }^{1}$, Liangcheng Zheng ${ }^{1}$

${ }^{1}$ Department of Thoracic Surgery, The First Affiliated Hospital of Wenzhou Medical University, Wenzhou 325000, Zhejiang Province, China.

Department of Thoracic Surgery, The First Affiliated Hospital of Wenzhou Medical University, Wenzhou 325000, Zhejiang Province, China.

Email address: 48936053@qq.com

Background. ceRNAs (competing endogenous RNAs) are a newly identified type of regulatory RNA. Accumulating evidence suggests that ceRNAs play an important role in the pathogenesis of diseases such as cancer. Thus, ceRNA dysregulation may represent an important molecular mechanism underlying cancer progression and poor prognosis. In this study, we aimed to identify ceRNAs that may serve as potential biomarkers for early diagnosis of lung

Methods. We performed differential gene expression analysis on TCGA-LUAD datasets to identify differentially expressed (DE) mRNAs, lncRNAs, and miRNAs at different tumor stages. Based on the ceRNA hypothesis and considering the synergistic or feedback regulation of ceRNAs, a lncRNA-miRNA-mRNA network was constructed. Functional analysis was performed using GO term and KEGG pathway enrichment analysis and KOBAS 2.0 software. Transcription factor analysis was carried out to identify direct targets of the transcription factors associated with LUAD prognosis. Identified differentially expressed genes were validated using

Results. Based on analysis of TCGA- LUAD datasets, we obtained 2610 DE mRNAs, 915 lncRNAs, and 125 miRNAs that were common to different tumor stages $\left(\mid \log _{2}\right.$ (Fold change) $\mid \geq 1$, FDR $<0.01$ ), respectively. Functional analysis showed that the aberrantly expressed mRNAs were closely related to tumor development. Survival analyses of the constructed ceRNA network modules demonstrated that five of them exhibit prognostic significance. The five ceRNA 
35

36

interaction modules contained one lncRNA (FENDRR), three mRNAs (EPAS1, FOXF1, and EDNRB), and four miRNAs (hsa-miR-148a, hsa-miR-195, hsa-miR-196b, and hsa-miR-301b).

The aberrant expression of one lncRNA and three mRNAs was verified in the LUAD GEO dataset. Transcription factor analysis demonstrated that EPAS1 directly targeted 13 DE mRNAs. Conclusion. Our observations indicate that lncRNA-related ceRNAs and TFs play an important role in LUAD. The present study provides novel insights into the molecular mechanisms underlying LUAD pathogenesis. Furthermore, our study facilitates the identification of potential biomarkers for the early diagnosis and prognosis of LUAD and therapeutic targets for its treatment.

\section{Introduction}

Lung cancer, which is one of the most common malignancies worldwide, poses a serious threat to human health and life (Jemal et al. 2011; Torre et al. 2015). In recent years, there has been an increase in the morbidity of lung cancer owing to deterioration of the environment (Cui et al. 2017; David et al. 2016; Haugen et al. 2000; Jin et al. 2014; Kooperstein et al. 1965; Weiss 1983). Furthermore, lung cancer is difficult to diagnose early and associated with poor prognosis and low survival rate (Bernaudin 2010; Hardavella et al. 2016; Onganer et al. 2005; Prim et al. 2010; Reinmuth et al. 2014; Tamura et al. 2015). The prognosis of patients with lung cancer is not only related to its biological factors and immune status, but is also closely related with the therapeutic factors (Fan et al. 2014; Prim et al. 2010; Ringbaek et al. 1999; Truong et al. 2011); association between multiple factors leads to complex changes in the disease and makes prognosis difficult. Tumor staging is an important factor in determining prognosis, including survival in cancer patients (Gong et al. 2017; Li et al. 2017). Studies have shown that patients with late stage lung cancer display extremely poor prognosis, with invasion and metastasis, and additionally respond poorly to clinical treatment (Mattern et al. 2002; Prim et al. 2010). Therefore, early detection of cancer should enable administration of effective treatment and considerably improve patient prognosis. Although the prognosis of non-small cell lung cancer (NSCLC) has been greatly improved following the introduction of multidisciplinary treatment, the prognosis of lung cancer patients and treatment efficiency remain poor (Fan et al. 2014; Huang et al. 2012; Miura et al. 1996; Pujol et al. 1994); therefore, the identification of biological markers and therapeutic targets for the early detection and treatment of recurrent lung tumor is critical.

Competing endogenous RNAs (ceRNAs) are a group of regulatory RNA molecules that compete with other RNA molecules to bind specific miRNAs, thereby regulating target gene expression 
70 (Phelps et al. 2016; Shukla et al. 2011; Tay et al. 2011). Many studies have suggested that

71 miRNA-mediated ceRNA regulatory mechanisms play crucial roles in tumor occurrence and

72 development (Bartel 2009; Hansen et al. 2013; Tay et al. 2014). Therefore, the identification of

73 ceRNA biomarkers related to lung cancer should enable the development of effective strategies

74 for the diagnosis and treatment of lung cancer.

75

76

77

78

79

80

81

82

83

84

85

86

87

88

89

90

91

92

93

94

95

96

97

98

99

100

101

102

103

In this study, we aimed to identify differentially expressed genes (DEGs) associated with lung adenocarcinoma (LUAD) development and construct a ceRNA regulatory network using the TCGA-LUAD dataset. Further, we examined the relationship of the identified genes and ceRNA interaction modules with overall survival and prognosis. Through our study, we sought to gain new insights into the molecular mechanisms underlying LUAD and identify potential biomarkers for early diagnosis of this disease.

\section{Materials \& Methods}

\section{Data source and preprocessing}

Lung adenocarcinoma (LUAD) RNA-Seq and lncRNA gene expression level3 data were obtained from the database Cancer Genome Atlas (TCGA, https://tcga-data.nci.nih.gov/tcga/).

After an initial filter (excluding samples with uncertain tumor stage), 569 samples were included for further study. Patient clinical data, including outcome and staging information, were also downloaded. Of the 569 samples, 510 were LUAD samples (168 with T1 stage, 276 with T2 stage, 47 with T3 stage, and 19 with T4 stage) and 59 were normal samples.

\section{Screening of genetically altered genes}

Gene raw read counts were used to perform differential expression analysis with DESeq2 (v 1.18.1) (Love et al. 2014), a R package that uses a model based on the negative binomial distribution and is widely used for RNA-seq data differential analysis. DEGs were defined by two criteria: false discovery rate $(\mathrm{FDR})<0.01$ and fold change $(\mathrm{FC}) \geq 2$. In order to filter out the low-quality DEGs, we performed the following additional filter strategy. For mRNA and miRNA, we only retained DEGs that showed FPKM or RPKM larger than the threshold of 1 for at least $10 \%$ samples; for lncRNA DEGs, the threshold was set to 0.1 . We then compared the normal tissue sample with different tumor stage samples (T1, T2, T3, and T4) to identify common DEGs for further analysis.

\section{Construction of IncRNA-associated ceRNA network (gene set construction)}


104

105

106

107

108

109

110

111

112

113

114

115

116

117

118

119

120

121

122

123

124

125

126

127

128

129

130

131

132

133

134

135

Based on the relationship between lncRNA, mRNA, miRNA, a ceRNA network was constructed using the following steps: 1) Both up-regulated and down-regulated LUAD-specific RNAs were chosen to construct the ceRNA network; 2) miRanda software was used to predict the miRNAlncRNA interactions, and interactions in starBase (Li et al. 2014) were also included; 3) miRNAmRNA interactions in miRTarbase and starBase were used; 4) Correlation between LUADspecific lncRNA and mRNA was calculated with WGCNA (Zhang \& Horvath 2005) corAndPvalue function and correlation pairs with coefficient $>0.8$ and $p$-value $<0.05$ were retained; 5) Hypergeometric method was used to test the enrichment significance of miRNAs between mRNA and lncRNA. The enrichment significance was calculated by using the following formula:

$$
P=\sum_{i=c}^{\min (K, n)} \frac{\left(\begin{array}{l}
K \\
i
\end{array}\right)\left(\begin{array}{l}
N-K \\
n-i
\end{array}\right)}{\left(\begin{array}{l}
N \\
n
\end{array}\right)}
$$

In the above formula, $\mathrm{N}$ represents the total number of miRNAs, $\mathrm{K}$ is the number of miRNAs targeting mRNA, $\mathrm{n}$ is the number of miRNAs targeting $\operatorname{lncRNA}$, and $\mathrm{c}$ is the number of miRNAs shared by the mRNA and lncRNA. Only ceRNA modules with FDR $<0.05$ were considered.

\section{Gene functional enrichment analysis}

In order to investigate key mRNAs at molecular and functional level, Gene Ontology (GO) and Kyoto Encyclopedia of Genes and Genomes (KEGG) pathway function enrichment analysis was performed. GOseq, which uses Wallenius' noncentral hypergeometric distribution model taking gene length bias into account, was used to perform GO enrichment analysis. GO terms with $p$ value less than 0.05 were considered significantly enriched. KOBAS 2.0 software was used to test statistical enrichment in KEGG pathways, and those with a Fisher's exact test $p$-value of less than 0.05 was considered to be significantly enriched (Xie et al. 2011).

\section{Transcription factor (TF) analysis}

Co-expression networks of LUAD-mRNA expression data sets were generated using Genie3 with EPAS1 and FOXF1 as TFs of interest. A threshold of 0.005 was used to filter the coexpression network. Additionally, Spearman correlations between the TFs and LUAD-specific mRNAs were calculated, and TF-mRNA pairs with coefficient $>0.03$ were retained as this value represents positive correlation. Using this procedure, we obtained a TF co-expression draft regulatory network. As the analysis was only based on co-expression, the results may include numerous indirect targets (such as downstream effects). To identify genes that are most likely the 
136 direct targets, we used RcisTarget (Aibar et al. 2017; Imrichova et al. 2015) to perform cis137 regulatory motif analysis of each TF regulon. RcisTarget is an R-package that identifies TF

138 binding motifs that are over-represented in a gene list. We used the database, scoring $500 \mathrm{bp}$ 139 upstream of TSS, and only motifs with a Normalized Enrichment Score (NES) > 3.0 were 140 considered associated with TFs of interest.

141

\section{Validation of gene expression with GEO data}

In order to validate the key differentially expressed molecules, we examined four datasets from among the Gene Expression Omnibus (GEO) datasets. Of the selected datasets, the GSE10072 study contained 58 LUAD tumor and 49 non-tumor tissue mRNA expression data, GSE32863 contained 58 LUAD tumor and 58 non-tumor tissue mRNA expression data, GSE85716 contained 6 LUAD tumor and 6 non-tumor tissue lncRNA expression data, and GSE104854 contained 3 LUAD (pooled from 9 tissues) and 3 non-tumor (pooled from 9 tissues) expression data. GSE10072, GSE32863, and GSE85716 datasets, which were derived from microarray data, were analyzed with GEO2R online. The GSE104854 dataset, which was obtained by high throughput sequencing, was analyzed with DESeq2 R package based on read counts.

\section{Statistical analysis}

To investigate the impact of the expression level of RNAs on the prognostic survival of patients, survival analysis was performed. In the analysis, Kaplan-Meier survival analyses and log-rank test were performed to study the relationship between RNA expression states (cutoff point: median value) and survival time. Univariate cox proportional hazards regression was applied to identify the RNAs associated with overall survival. In order to evaluate the potential of dysregulated ceRNA modules as biomarkers, the risk scoring classifier was constructed. For each ceRNA module, we calculated the risk score for multivariate survival analysis to determine the prognostic influence of the RNAs as a whole. All statistical analyses were performed using R version 3.4.3 software.

\section{Results}

\section{Differentially expressed RNAs in LUAD} miRNAs were extracted from TCGA and analyzed with R package (DESeq2). In this analysis, we compared adjacent normal lung tissue with lung cancer subtypes defined by pathology stage (T1-T4), respectively. When we combined these four groups and analyzed for differentially expressed RNAs, 2160 mRNAs (1527 up- and 1083 down-regulated), 915 lncRNAs (662 up- 
171 and 253 down-regulated), and 125 miRNAs (73 up- and 52 down-regulated) showed consistently

172 differential expression (fold change $\geq 2$, FDR $<0.01$ ) (Fig.1 A-C). Fig. 2 A-C shows the

173 heatmap of the common DEGs; the lung tumor tissue samples could be easily distinguished from

174 the adjacent non-tumor lung tissues from the heat map. Based on these data, differentially

175 expressed mRNAs, lncRNAs, and miRNAs were selected for further analysis.

176

177

Functional enrichment of mRNA DEGs

178

In order to understand the functions of aberrantly expressed genes in this study, a total of 2610

179 aberrantly expressed genes were analyzed. We used GOseq for GO annotation and KOBAS 2.0, which includes the Kyoto Encyclopedia of Genes and Genomes (KEGG) database, to classify and analyze the potential gene functions in the pathways. Our analysis revealed an enrichment of 2879 GO terms and 30 KEGG pathways ( $p$ value $<0.05$ ) (Table S1). GO analyses were classified into three functional groups: molecular function group, biological process group, and cellular component group (Fig. 3). Enrichment of these DEGs represents a measure of significance of a function. As shown in Fig. 3, the most enriched GO biological process terms were "single-organism cellular process" and "single-multicellular organism process". Other significant GO terms included "cell proliferation", "cell adhesion", "system development", "cell migration", and "cell adhesion". The significant pathways identified by KEGG pathway analysis included viral carcinogenesis, systemic lupus erythematosus, cell adhesion molecules, and p53 signaling pathway (Fig. 4). Our analysis indicated that 2610 dysregulated mRNAs were involved in signaling pathways related to environmental information processing and human diseases such as cancer.

193

\section{ceRNA network analysis and integrated ceRNA network construction}

Based on the miRNAs and mRNAs described in Fig. 2, starBase v2.0 database and miRanda were used to predict miRNA-targeted RNAs. Then, combing miRNA-lncRNA interactions with the miRNA-mRNA interactions, an integrated lncRNA-miRNA-mRNA ceRNA network was established. As shown in Fig. 5, the network contained 107 interactions, and included 5 lncRNAs, 20 mRNAs, and 34 miRNAs (Table S2). After ranking the molecules according to their degree, FENDRR, which is a lncRNA, showed the highest degree (degree = 38). FOXF1, $E D N R B$, and EPAS1 are all mRNA molecules, ranking the second, third and fourth respectively. In other studies, FENDRR was demonstrated to be down-regulated in breast and gastric cancers and to participate in regulating tumor malignancy (Li et al. 2018; Xu et al. 2014). FOXF1 and EPAS1 are TFs that have been demonstrated to participate in tumor progression (Putra et al. 
206

207

208

209

210

211

212

213

214

215

216

217

218

219

220

221

222

223

224

225

226

227

228

229

230

231

232

233

234

235

236

237

238

239

240

241

phosphatidylinositol-calcium second messenger system, and is also closely related with cancer (Chen et al. 2013; Wuttig et al. 2012).

\section{Key ceRNAs and their association with clinical features}

In order to further explore the key role of ceRNAs in the occurrence and development of LUAD, we aimed to identify prognostic-specific ceRNAs. We calculated expression of each ceRNA and performed prognosis analyses (e.g. of overall survival time) to determine the survival-significant lncRNA-miRNA-mRNA interactions based on Cox analysis $(P$ value $<0.05)$. As presented in Fig. 6 and Table 1, univariate Cox regression analysis showed that EDNRB-FENDRR-hsa-mir196b, EPAS1-FENDRR-hsa-mir-148a, FOXF1-FENDRR-hsa-mir-148a, FOXF1-FENDRR-hsamir-195, and FOXF1-FENDRR-hsa-mir-301b ceRNA interactions were associated with the overall survival of patients with LUAD. Kaplan-Meier survival curves indicated that EDNRBFENDRR-hsa-mir-196b interaction was negatively correlated with overall survival, whereas EPAS1-FENDRR-hsa-mir-148a, FOXF1-FENDRR-hsa-mir-148a, FOXF1-FENDRR-hsa-mir195, and FOXF1-FENDRR-hsa-mir-301b were positively correlated with survival.

\section{TF cis-target analysis}

TFs play important roles in the regulation of biological processes by binding enhancer or promoter regions of DNA, thereby activating or suppressing gene expression. This allows genes to be expressed in the right cell at the appropriate time. In this study, we found that EPAS1 and FOXF1 showed a high degree of representation in the ceRNA network. This finding suggests that these two TFs play an important role in LUAD and may be regulated by a ceRNA-based mechanism. In order to verify the direct targets of the two TFs, we analyzed them further. Coexpressed genes may be regulated by the same or similar transcription factors; however, they may include numerous indirect targets. Therefore, we performed binding motif enrichment analysis to identify direct target genes of the two TFs. In this study, we use GENIE3 to perform TF-genes co-expression analysis and RcisTarget to analyze enriched motifs in the TSS upstream of the gene-sets. As shown in Fig.7, the EPAS1 co-expressed gene-set was enriched for the“cisbp_M6212" motif (NES = 3.21); likely direct gene targets of EPAS1 include ADRB2, CALCRL, EMP2, FHL1, GRIA1, LGI3, LIMS2, NCKAP5, RXFP1, SLC6A4, SMAD6, STX11, and TMEM100. However, the FOXF1 co-expressed gene-set did not show enrichment for any motifs.

\section{Validation of the expression of key RNA molecules with GEO data}

Finally, four reported studies were screened out from the GEO to verify the differential expression of key mRNAs and lncRNAs in LUAD. GSE10072 and GSE32863 were used for EPAS1, FOXF1, and EDNRB mRNA verification. GSE85716 and GSE104854 were used for 
242 FENDRR differential expression. As shown in Fig. 8 and Table 2, all four molecules showed

243 significantly lower levels of expression in tumor datasets, which is consistent with the results of

244 our study.

245

\section{Discussion}

247 NSCLC, which mainly includes adenocarcinoma and squamous cell carcinoma, accounts for 248 about one-sixth of cancer deaths in the global population (Jemal et al. 2011; Torre et al. 2015).

249 Despite numerous advances in the treatment of lung cancer, the prognosis of this disease remains 250 poor, and the 5-year overall survival rate is only 11\% (Huang et al. 2012; Miura et al. 1996).

251 Therefore, elucidation of the underlying mechanisms is indispensable for diagnosing, preventing, 252 and treating NSCLC.

253

254

255

256

257

258

259

260

261

262

263

264

265

266

267

268

269

270

271

272

273

274

275

276

With the development of high-throughput sequencing technology, accumulating omics data reveal that the occurrence and development of the disease is closely related to multiple factors, including genomic mutations, expression profiles, copy number variants (CNV), and structural variations (SV) (Balbin et al. 2013; Kikutake \& Yahara 2016; Kim et al. 2015; Yan et al. 2017). In recent years, several studies have found that multiple RNA molecules are closely related to tumor progression (Chen et al. 2018; He et al. 2018; Men et al. 2018; Shang et al. 2017; Song et al. 2018; Tang et al. 2017; Wang et al. 2018; Yu et al. 2017). For example, lncRNA-DANCR enhances the stemness feature of cancer cells to increase the degree of malignancy of lung cancer (Lu et al. 2018; Zhen et al. 2018); miRNA-221 was reported to regulate the process of tumor differentiation, proliferation, migration, and invasion (Lv et al. 2014; Yin et al. 2017). These RNA molecules represent biological markers for the prognosis of lung cancer. In this study, we found that multiple novel ceRNA molecules can be used as novel prognostic markers; further, we examined the putative molecular mechanisms underlying the development of tumors.

miRNAs modulate the expression of target genes by regulating the transcription and stability of their mRNAs or lncRNAs. Investigation of the lncRNA-mRNA co-expression network is important for analysis of the function and regulatory mechanisms of lncRNAs (Fang et al. 2018). In this study, analysis showed that a number of mRNAs, IncRNAs, and miRNAs that were differentially expressed were common at each stage of lung cancer. GO and KEGG analysis showed that the DEGs were mainly involved in "single-organism cellular process", "singlemulticellular organism process", "cell proliferation", "cell adhesion", "system development", "cell migration", and "cell adhesion biological processes". Further analysis revealed that the upregulated genes were involved in the mitotic cell cycle and nuclear division process (Table S3), 
277 while down-regulated genes were involved in vasculature development, cell motility, cell 278 migration, and cell proliferation (Table S4). KEGG analysis found that the up-regulated genes 279 were mainly involved in cancer and other related signaling pathways such as the p53 signaling 280 pathway.

281

282

283

284

285

286

287

288

289

290

291

292

293

294

295

296

297

298

299

300

301

302

303

304

305

306

307

308

309

310

311

Survival curve analysis reveals the correlation between gene expression and patient prognosis. Our study found that the five most important ceRNA-related RNA molecules were significantly correlated with the survival rate of patients with lung cancer $(P<0.01)$, and most of the genes were highly correlated with cancer. The five ceRNA interactions consisted of one lncRNA, three mRNAs, and four miRNAs. The lncRNA, FENDRR (Grote et al. 2013), which is produced from a spliced long non-coding RNA transcribed bidirectionally with FOXF1 on the opposite strand, is be down-regulated in both breast cancer and gastric cancer. In breast cancer, lower expression level of FENDRR was associated with shorter overall survival and progression-free survival in breast cancer patients. FENDRR knockdown promoted breast cancer cell proliferation and migration, and suppressed cell apoptosis; in contrast, its overexpression inhibited tumor growth in a xenograft model ( $\mathrm{Li}$ et al. 2018). FENDRR was also reported to be down-regulated in gastric cancer cell lines and tissues. Further, FENDRR expression was negatively correlated with tumor metastasis and stage (Xu et al. 2014). In our study, FENDRR was also down-regulated in LUAD, and showed the highest degree in ceRNA network, indicating its important role in LUAD. The three mRNAs, FOXF1, EDNRB, and EPAS1, ranked $2^{\text {nd }}, 3^{\text {rd }}$, and $4^{\text {th }}$, respectively in the ceRNA network. FOXF1 and EPAS1 are both TFs that have been associated with tumor malignancy (Putra et al. 2015; Tamura et al. 2014). Our TF analysis indicated that EPAS1 directly regulates 13 molecules associated with LUAD, namely ADRB2, CALCRL, EMP2, FHL1, GRIA1, LGI3, LIMS2, NCKAP5, RXFP1, SLC6A4, SMAD6, STX11, and TMEM100. EDNRB, a G proteincoupled receptor, is also closely related with tumor (Chen et al. 2013; Wuttig et al. 2012). Our findings suggest that these molecules represent potential cancer-related biomarkers.

\section{Conclusions}

In summary, in the present study, we comprehensively analyzed the expression of key RNA molecules in the progression of lung cancer and identified key RNA molecules (including mRNA/lncRNA/miRNA) that were abnormally expressed in different stages of this disease. Further, using the above-mentioned key RNA molecules to construct a ceRNA-gene interaction network, it was found that several FOXF1- and EDNRB-related ceRNA molecules play an important role in the development and progression of lung cancer, and can affect the prognosis of this disease. This series of related ceRNA molecules is expected to provide a novel basis for 
312 diagnosis and evaluation of prognosis, and represents potential new targets for the treatment of

313 lung cancer.

314

\section{Acknowledgments}

316 The authors would like to thank TCGA for providing the data and Mrs. Gu JY for her technical 317 assistance.

318

319

\section{Data Availability}

320

321

322

323

324

325

326

327

328

329

330

331

332

333

334

335

336

337

338

339

340

341

342

343

344

345

The following information was supplied regarding data availability:

The origin RNAseq data used in our study were all downloaded from the TCGA data portal: https://portal.gdc.cancer.gov/;

GEO data link of mRNA: https://www.ncbi.nlm.nih.gov/geo/query/acc.cgi?acc=GSE10072; https:/www.ncbi.nlm.nih.gov/geo/query/acc.cgi?acc=GSE32863;

GEO data link of lncRNA: https://www.ncbi.nlm.nih.gov/geo/query/acc.cgi?acc=GSE85716;

https://www.ncbi.nlm.nih.gov/geo/query/acc.cgi?acc=GSE104854.

\section{Ethics approval and consent to participate}

Not applicable.

\section{Patient consent for publication}

Not applicable.

\section{References}

Aibar S, Gonzalez-Blas CB, Moerman T, Huynh-Thu VA, Imrichova H, Hulselmans G, Rambow F, Marine JC, Geurts P, Aerts J, van den Oord J, Atak ZK, Wouters J, and Aerts S. 2017. SCENIC: single-cell regulatory network inference and clustering. Nat Methods 14:1083-1086. 10.1038/nmeth.4463

Balbin OA, Prensner JR, Sahu A, Yocum A, Shankar S, Malik R, Fermin D, Dhanasekaran SM, Chandler B, Thomas D, Beer DG, Cao X, Nesvizhskii AI, and Chinnaiyan AM. 2013. Reconstructing targetable pathways in lung cancer by integrating diverse omics data. Nat Commun 4:2617. 10.1038/ncomms3617

Bartel DP. 2009. MicroRNAs: target recognition and regulatory functions. Cell 136:215-233. 10.1016/j.cell.2009.01.002

Bernaudin JF. 2010. [Molecular characteristics of lung cancer]. Bull Cancer 97:1323-1335. 
346

347

348

349

350

351

352

353

354

355

356

357

358

359

360

361

362

363

364

365

366

367

368

369

370

371

372

373

374

375

376

377

378

379

380

381

10.1684/bdc.2010.1211

Chen C, Wang L, Liao Q, Huang Y, Ye H, Chen F, Xu L, Ye M, and Duan S. 2013.

Hypermethylation of EDNRB promoter contributes to the risk of colorectal cancer. Diagn Pathol 8:199. 10.1186/1746-1596-8-199

Chen J, Wang S, Jia S, Ding G, Jiang G, and Cao L. 2018. Integrated Analysis of Long NonCoding RNA and mRNA Expression Profile in Pancreatic Cancer Derived Exosomes Treated Dendritic Cells by Microarray Analysis. J Cancer 9:21-31. 10.7150/jca.21749

Cui JJ, Wang LY, Zhu T, Gong WJ, Zhou HH, Liu ZQ, and Yin JY. 2017. Gene-gene and geneenvironment interactions influence platinum-based chemotherapy response and toxicity in non-small cell lung cancer patients. Sci Rep 7:5082. 10.1038/s41598-017-05246-8

David SP, Wang A, Kapphahn K, Hedlin H, Desai M, Henderson M, Yang L, Walsh KM, Schwartz AG, Wiencke JK, Spitz MR, Wenzlaff AS, Wrensch MR, Eaton CB, Furberg H, Mark Brown W, Goldstein BA, Assimes T, Tang H, Kooperberg CL, Quesenberry CP, Tindle H, Patel MI, Amos CI, Bergen AW, Swan GE, and Stefanick ML. 2016. Gene by Environment Investigation of Incident Lung Cancer Risk in African-Americans. EBioMedicine 4:153-161. 10.1016/j.ebiom.2016.01.002

Fan X, Zhang Z, and Liu Y. 2014. Therapeutic efficacy and prognosis in the treatment of lung cancer by video-assisted thoracoscopic surgery. Chin Med J (Engl) 127:2096.

Fang L, Wang H, and Li P. 2018. Systematic analysis reveals a lncRNA-mRNA co-expression network associated with platinum resistance in high-grade serous ovarian cancer. Invest New Drugs 36:187-194. 10.1007/s10637-017-0523-3

Gong B, Li Y, Cheng Z, Wang P, Luo L, Huang H, Duan S, and Liu F. 2017. GRIK3: A novel oncogenic protein related to tumor TNM stage, lymph node metastasis, and poor prognosis of GC. Tumour Biol 39:1010428317704364. 10.1177/1010428317704364

Grote P, Wittler L, Hendrix D, Koch F, Wahrisch S, Beisaw A, Macura K, Blass G, Kellis M, Werber M, and Herrmann BG. 2013. The tissue-specific lncRNA Fendrr is an essential regulator of heart and body wall development in the mouse. Dev Cell 24:206-214. 10.1016/j.devcel.2012.12.012

Hansen TB, Jensen TI, Clausen BH, Bramsen JB, Finsen B, Damgaard CK, and Kjems J. 2013. Natural RNA circles function as efficient microRNA sponges. Nature 495:384-388. 10.1038/nature11993

Hardavella G, George R, and Sethi T. 2016. Lung cancer stem cells-characteristics, phenotype. Transl Lung Cancer Res 5:272-279. 10.21037/tlcr.2016.02.01

Haugen A, Ryberg D, Mollerup S, Zienolddiny S, Skaug V, and Svendsrud DH. 2000. Geneenvironment interactions in human lung cancer. Toxicol Lett 112-113:233-237.

He ZH, Qin XH, Zhang XL, Yi JW, and Han JY. 2018. Long noncoding RNA GIHCG is a 
382

383

384

385

386

387

388

389

390

391

392

393

394

395

396

397

398

399

400

401

402

403

404

405

406

407

408

409

410

411

412

413

414

415

416

417

potential diagnostic and prognostic biomarker and therapeutic target for renal cell carcinoma. Eur Rev Med Pharmacol Sci 22:46-54. 10.26355/eurrev_201801_14099

Huang Y, Liu D, Chen B, Zeng J, Wang L, Zhang S, Mo X, and Li W. 2012. Loss of Bad expression confers poor prognosis in non-small cell lung cancer. Med Oncol 29:16481655. 10.1007/s12032-011-0060-4

Imrichova H, Hulselmans G, Atak ZK, Potier D, and Aerts S. 2015. i-cisTarget 2015 update: generalized cis-regulatory enrichment analysis in human, mouse and fly. Nucleic Acids Res 43:W57-64. 10.1093/nar/gkv395

Jemal A, Bray F, Center MM, Ferlay J, Ward E, and Forman D. 2011. Global cancer statistics. CA Cancer J Clin 61:69-90. 10.3322/caac.20107

Jin S, Deng Y, Hao JW, Li Y, Liu B, Yu Y, Shi FD, and Zhou QH. 2014. NK cell phenotypic modulation in lung cancer environment. PLoS One 9:e109976. 10.1371/journal.pone.0109976

Kikutake C, and Yahara K. 2016. Identification of Epigenetic Biomarkers of Lung Adenocarcinoma through Multi-Omics Data Analysis. PLoS One 11:e0152918. 10.1371/journal.pone.0152918

Kim S, Herazo-Maya JD, Kang DD, Juan-Guardela BM, Tedrow J, Martinez FJ, Sciurba FC, Tseng GC, and Kaminski N. 2015. Integrative phenotyping framework (iPF): integrative clustering of multiple omics data identifies novel lung disease subphenotypes. $B M C$ Genomics 16:924. 10.1186/s12864-015-2170-4

Kooperstein SI, Schifrin A, and Leahy TJ. 1965. Lung cancer and environment: a study of longterm exposure to motor fumes in tunnels. J Occup Med 7:626-629.

Li B, Cui Y, Diehn M, and Li R. 2017. Development and Validation of an Individualized Immune Prognostic Signature in Early-Stage Nonsquamous Non-Small Cell Lung Cancer. JAMA Oncol 3:1529-1537. 10.1001/jamaoncol.2017.1609

Li JH, Liu S, Zhou H, Qu LH, and Yang JH. 2014. starBase v2.0: decoding miRNA-ceRNA, miRNA-ncRNA and protein-RNA interaction networks from large-scale CLIP-Seq data. Nucleic Acids Res 42:D92-97. 10.1093/nar/gkt1248

Li Y, Zhang W, Liu P, Xu Y, Tang L, Chen W, and Guan X. 2018. Long non-coding RNA FENDRR inhibits cell proliferation and is associated with good prognosis in breast cancer. Onco Targets Ther 11:1403-1412. 10.2147/OTT.S149511

Love MI, Huber W, and Anders S. 2014. Moderated estimation of fold change and dispersion for RNA-seq data with DESeq2. Genome Biol 15:550. 10.1186/s13059-014-0550-8

Lu QC, Rui ZH, Guo ZL, Xie W, Shan S, and Ren T. 2018. LncRNA-DANCR contributes to lung adenocarcinoma progression by sponging miR-496 to modulate mTOR expression. $J$ Cell Mol Med 22:1527-1537. 10.1111/jcmm.13420

Peer] reviewing PDF | (2018:10:32312:1:2:NEW 2 Feb 2019) 
418

419

420

421

422

423

424

425

426

427

428

429

430

431

432

433

434

435

436

437

438

439

440

441

442

443

444

445

446

447

448

449

450

451

452

453

Lv J, Xu L, Xu Y, Qiu M, Yang X, Wang J, Yin R, and Xu L. 2014. [Expression of MiRNA-221 in non-small cell lung cancer tissues and correlation with prognosis]. Zhongguo Fei Ai Za Zhi 17:221-225. 10.3779/j.issn.1009-3419.2014.03.07

Mattern J, Koomagi R, and Volm M. 2002. Characteristics of long-term survivors of untreated lung cancer. Lung Cancer 36:277-282.

Men CD, Liu QN, and Ren Q. 2018. A prognostic 11 genes expression model for ovarian cancer. J Cell Biochem 119:1971-1978. 10.1002/jcb.26358

Miura H, Kato H, Konaka C, Usuda J, Uchida O, and Taira O. 1996. Primary lung cancer of the middle lobe. Is its prognosis poor? Lung Cancer 14:273-279.

Onganer PU, Seckl MJ, and Djamgoz MB. 2005. Neuronal characteristics of small-cell lung cancer. Br J Cancer 93:1197-1201. 10.1038/sj.bjc.6602857

Phelps M, Coss C, Wang H, Cook M, Reproducibility Project: Cancer B, and Reproducibility Project Cancer B. 2016. Registered report: Coding-independent regulation of the tumor suppressor PTEN by competing endogenous mRNAs. Elife 5. 10.7554/eLife. 12470

Prim JM, Barcala FJ, Esquete JP, Reino AP, Lopez AF, and Cuadrado LV. 2010. Lung cancer in a health area of Spain: incidence, characteristics and survival. Eur J Cancer Care (Engl) 19:227-233. 10.1111/j.1365-2354.2008.01008.x

Pujol JL, Monnier A, Berille J, Cerrina ML, Douillard JY, Riviere A, Grandgirard A, Gouva S, Bizzari JP, and Le Chevalier T. 1994. Phase II study of nitrosourea fotemustine as singledrug chemotherapy in poor-prognosis non-small-cell lung cancer. Br J Cancer 69:11361140.

Putra AC, Eguchi H, Lee KL, Yamane Y, Gustine E, Isobe T, Nishiyama M, Hiyama K, Poellinger L, and Tanimoto K. 2015. The A Allele at rs13419896 of EPAS1 Is Associated with Enhanced Expression and Poor Prognosis for Non-Small Cell Lung Cancer. PLoS One 10:e0134496. 10.1371/journal.pone.0134496

Reinmuth N, Stumpf P, Stumpf A, Muley T, Kobinger S, Hoffmann H, Herth FJ, Schnabel PA, Bischoff $\mathrm{H}$, and Thomas M. 2014. Characteristics of lung cancer after a previous malignancy. Respir Med 108:910-917. 10.1016/j.rmed.2014.02.015

Ringbaek T, Borgeskov S, Lange P, and Viskum K. 1999. Diagnostic and therapeutic process and prognosis in suspected lung cancer. Scand Cardiovasc J 33:337-343.

Shang J, Song Q, Yang Z, Li D, Chen W, Luo L, Wang Y, Yang J, and Li S. 2017. Identification of lung adenocarcinoma specific dysregulated genes with diagnostic and prognostic value across 27 TCGA cancer types. Oncotarget 8:87292-87306. 10.18632/oncotarget.19823

Shukla GC, Singh J, and Barik S. 2011. MicroRNAs: Processing, Maturation, Target Recognition and Regulatory Functions. Mol Cell Pharmacol 3:83-92.

Song J, Xu Q, Zhang H, Yin X, Zhu C, Zhao K, and Zhu J. 2018. Five key lncRNAs considered 
454

455

456

457

458

459

460

461

462

463

464

465

466

467

468

469

470

471

472

473

474

475

476

477

478

479

480

481

482

483

484

485

486

487

488

489

as prognostic targets for predicting pancreatic ductal adenocarcinoma. J Cell Biochem 119:4559-4569. 10.1002/jcb.26598

Tamura M, Sasaki Y, Koyama R, Takeda K, Idogawa M, and Tokino T. 2014. Forkhead transcription factor FOXF1 is a novel target gene of the p53 family and regulates cancer cell migration and invasiveness. Oncogene 33:4837-4846. 10.1038/onc.2013.427

Tamura T, Kurishima K, Watanabe H, Shiozawa T, Nakazawa K, Ishikawa H, Satoh H, and Hizawa N. 2015. Characteristics of clinical N0 metastatic non-small cell lung cancer. Lung Cancer 89:71-75. 10.1016/j.lungcan.2015.04.002

Tang RX, Chen WJ, He RQ, Zeng JH, Liang L, Li SK, Ma J, Luo DZ, and Chen G. 2017. Identification of a RNA-Seq based prognostic signature with five lncRNAs for lung squamous cell carcinoma. Oncotarget 8:50761-50773. 10.18632/oncotarget.17098

Tay Y, Kats L, Salmena L, Weiss D, Tan SM, Ala U, Karreth F, Poliseno L, Provero P, Di Cunto F, Lieberman J, Rigoutsos I, and Pandolfi PP. 2011. Coding-independent regulation of the tumor suppressor PTEN by competing endogenous mRNAs. Cell 147:344-357. 10.1016/j.cell.2011.09.029

Tay Y, Rinn J, and Pandolfi PP. 2014. The multilayered complexity of ceRNA crosstalk and competition. Nature 505:344-352. 10.1038/nature12986

Torre LA, Bray F, Siegel RL, Ferlay J, Lortet-Tieulent J, and Jemal A. 2015. Global cancer statistics, 2012. CA Cancer J Clin 65:87-108. 10.3322/caac.21262

Truong MT, Viswanathan C, and Erasmus JJ. 2011. Positron emission tomography/computed tomography in lung cancer staging, prognosis, and assessment of therapeutic response. $J$ Thorac Imaging 26:132-146. 10.1097/RTI.0b013e3182128704

Wang W, Wu F, Zhao Z, Wang KY, Huang RY, Wang HY, Lan Q, Wang JF, and Zhao JZ. 2018. Long noncoding RNA LINC00152 is a potential prognostic biomarker in patients with high-grade glioma. CNS Neurosci Ther. 10.1111/cns.12850

Weiss W. 1983. Environment and type of lung cancer. Am Rev Respir Dis 128:1099-1100. 10.1164/arrd.1983.128.6.1099a

Wuttig D, Zastrow S, Fussel S, Toma MI, Meinhardt M, Kalman K, Junker K, Sanjmyatav J, Boll K, Hackermuller J, Rolle A, Grimm MO, and Wirth MP. 2012. CD31, EDNRB and TSPAN7 are promising prognostic markers in clear-cell renal cell carcinoma revealed by genome-wide expression analyses of primary tumors and metastases. Int J Cancer 131:E693-704. 10.1002/ijc.27419

Xie C, Mao X, Huang J, Ding Y, Wu J, Dong S, Kong L, Gao G, Li CY, and Wei L. 2011. KOBAS 2.0: a web server for annotation and identification of enriched pathways and diseases. Nucleic Acids Res 39:W316-322. 10.1093/nar/gkr483

Xu TP, Huang MD, Xia R, Liu XX, Sun M, Yin L, Chen WM, Han L, Zhang EB, Kong R, De W, 
490

491

492

493

494

495

496

497

498

499

500

501

502

503

504

505

506

507 and Shu YQ. 2014. Decreased expression of the long non-coding RNA FENDRR is associated with poor prognosis in gastric cancer and FENDRR regulates gastric cancer cell metastasis by affecting fibronectin1 expression. J Hematol Oncol 7:63. 10.1186/s 13045-014-0063-7

Yan H, Bi L, Wang Y, Zhang X, Hou Z, Wang Q, Snijders AM, and Mao JH. 2017. Integrative analysis of multi-omics data reveals distinct impacts of DDB1-CUL4 associated factors in human lung adenocarcinomas. Sci Rep 7:333. 10.1038/s41598-017-00512-1

Yin Z, Xu M, and Li P. 2017. miRNA-221 acts as an oncogenic role by directly targeting TIMP2 in non-small-cell lung carcinoma. Gene 620:46-53. 10.1016/j.gene.2017.04.007

Yu L, Fang F, Lu S, Li X, Yang Y, and Wang Z. 2017. IncRNA-HIT promotes cell proliferation of non-small cell lung cancer by association with E2F1. Cancer Gene Ther 24:221-226. $10.1038 /$ cgt.2017.10

Zhang B, and Horvath S. 2005. A general framework for weighted gene co-expression network analysis. Stat Appl Genet Mol Biol 4:Article17. 10.2202/1544-6115.1128

Zhen Q, Gao LN, Wang RF, Chu WW, Zhang YX, Zhao XJ, Lv BL, and Liu JB. 2018. LncRNA DANCR Promotes Lung Cancer by Sequestering miR-216a. Cancer Control 25:1073274818769849. 10.1177/1073274818769849 


\section{Figure 1}

Significantly expressed genes in LUAD

(A) The Venn diagram of mRNA DEGs, the circle represents mRNA DEGs between normal tissue and tumor stage tissue; (B) The Venn diagram of IncRNA DEGs; (C) The Venn diagram of miRNA DEGs

A

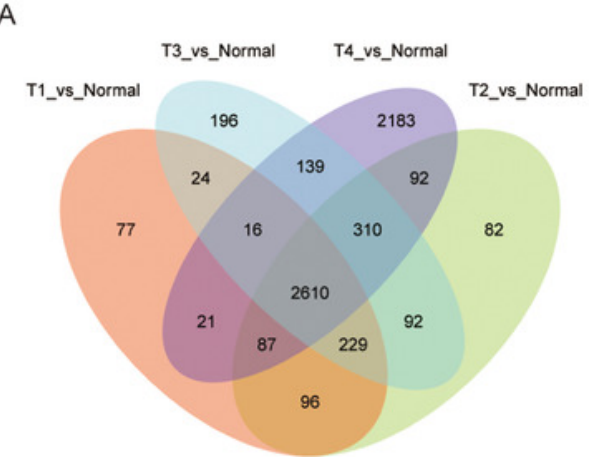

B

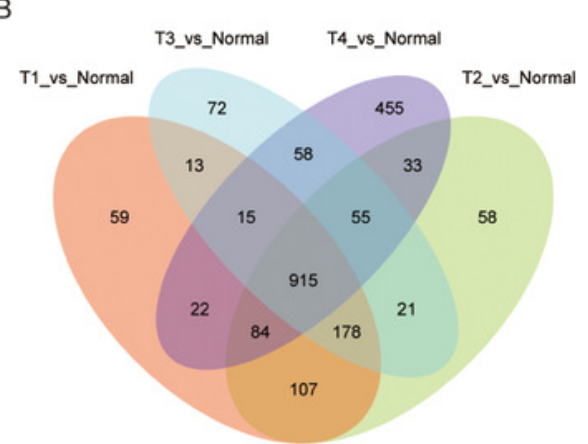

C

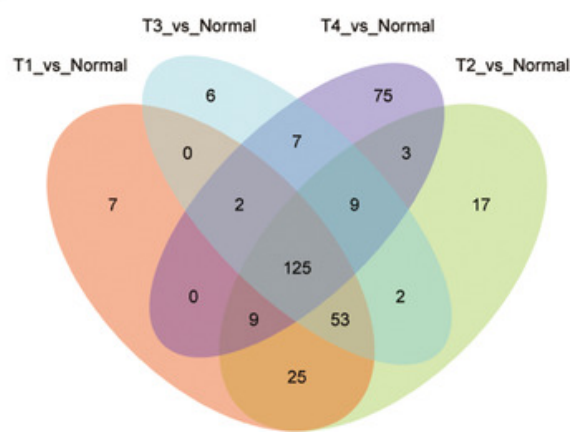


Figure 2

Heatmap of significantly expressed common genes between different pathology stages of LUAD

(A) mRNA DEGs; (B) IncRNA DEGs; (C) miRNA DEGs. a

A

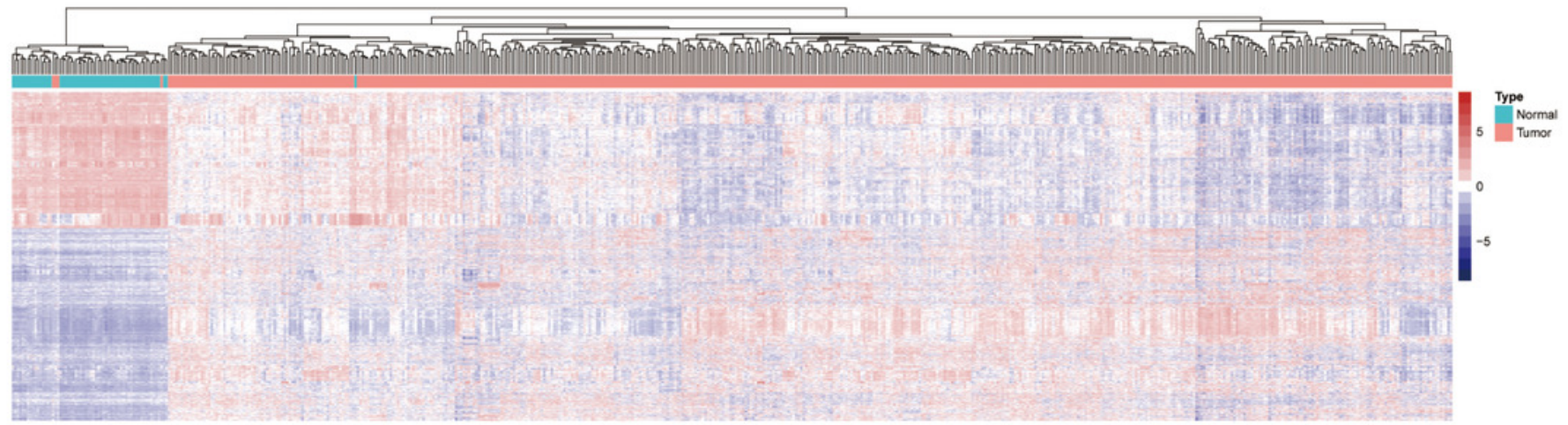

B

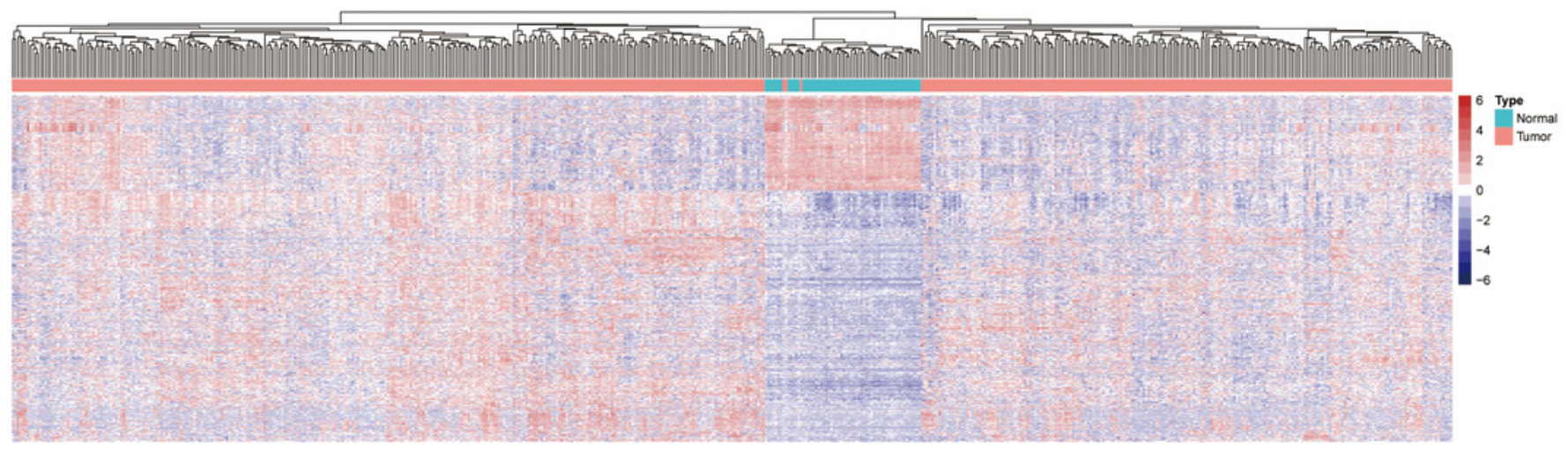

C

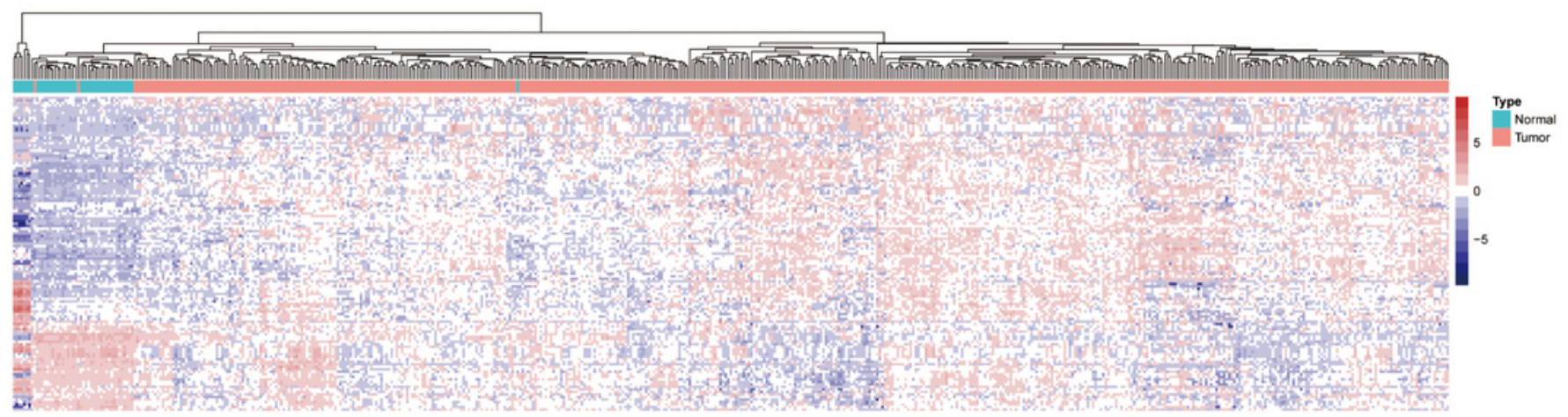


Figure 3

Gene Ontology analysis and significant enriched GO terms of DEGs in LUAD .

GO analysis classified the DEGs into 3 groups (i.e., molecular function, biological process and cellular component $\square$

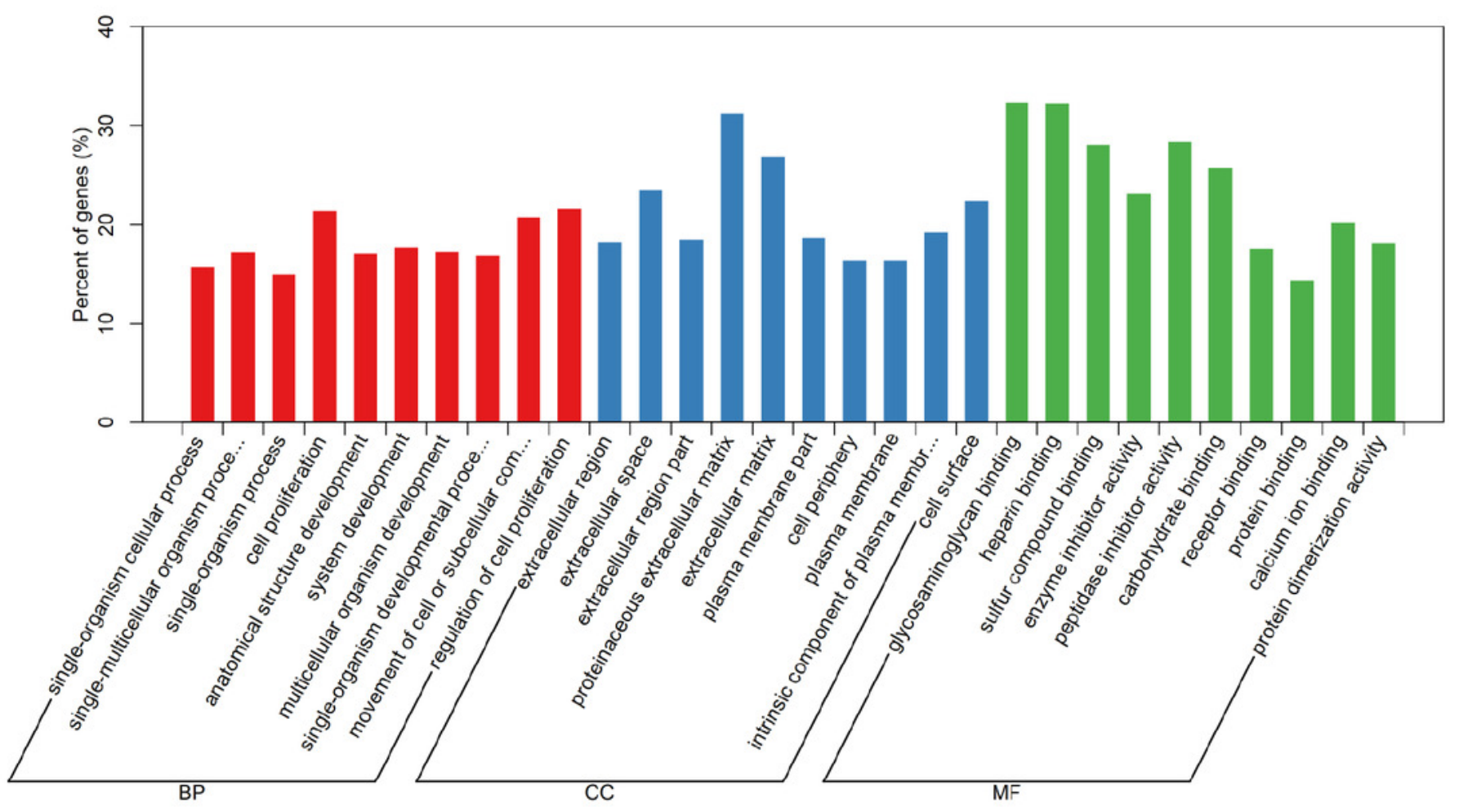




\section{Figure 4}

\section{Significantly enriched pathway terms in LUAD s and 5ST]}

\section{The functional and signaling pathway enrichment were conducted using KOBAS 2.0 software.}

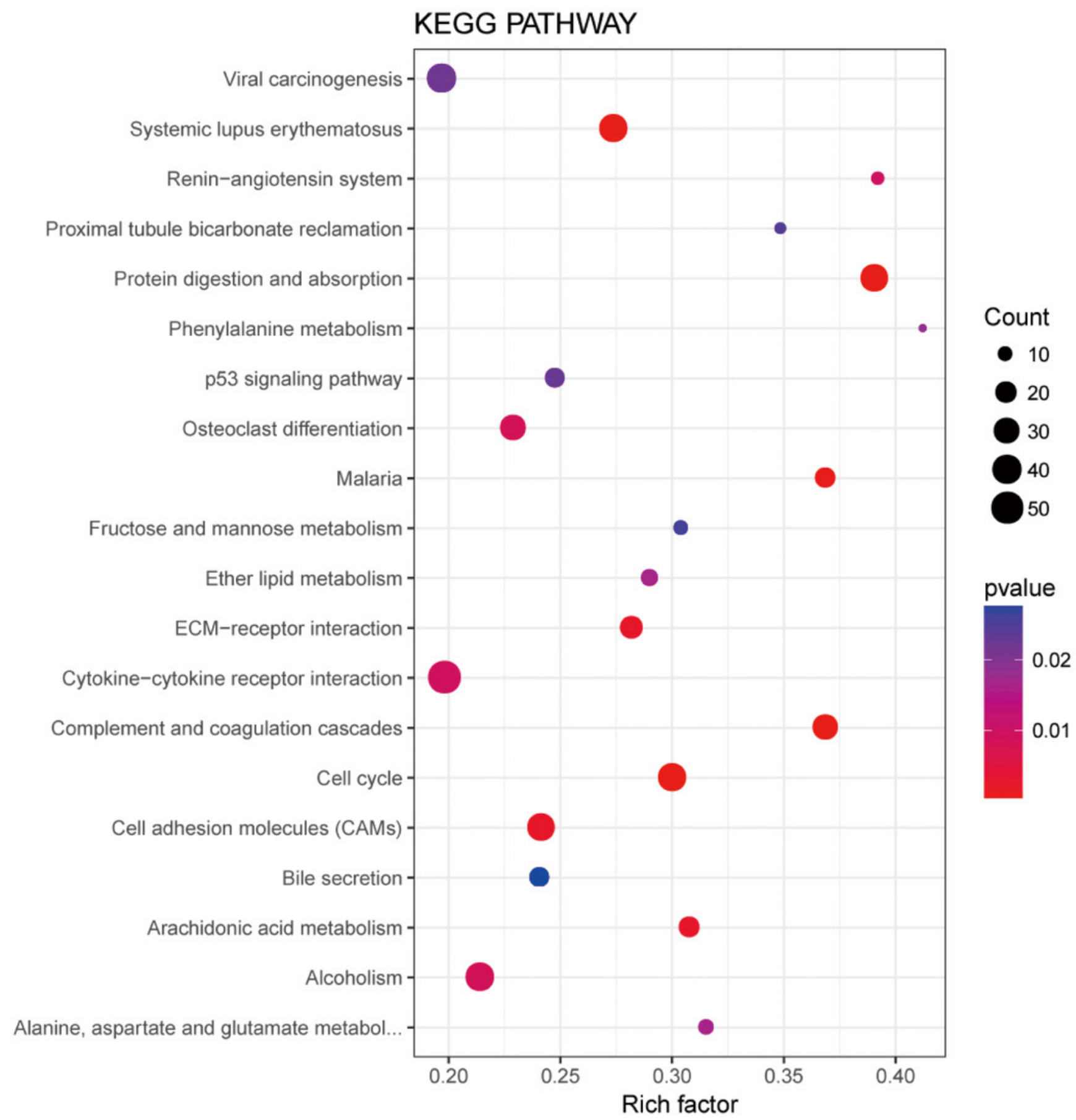


Figure 5

The IncRNA-miRNA-mRNA ceRNA network constructed from DEGs

Red represents up-regulated genes and yellow represents down-regulated genes. Circle represents mRNA, rectangle represents InCRNA, and triangle represents miRNA.

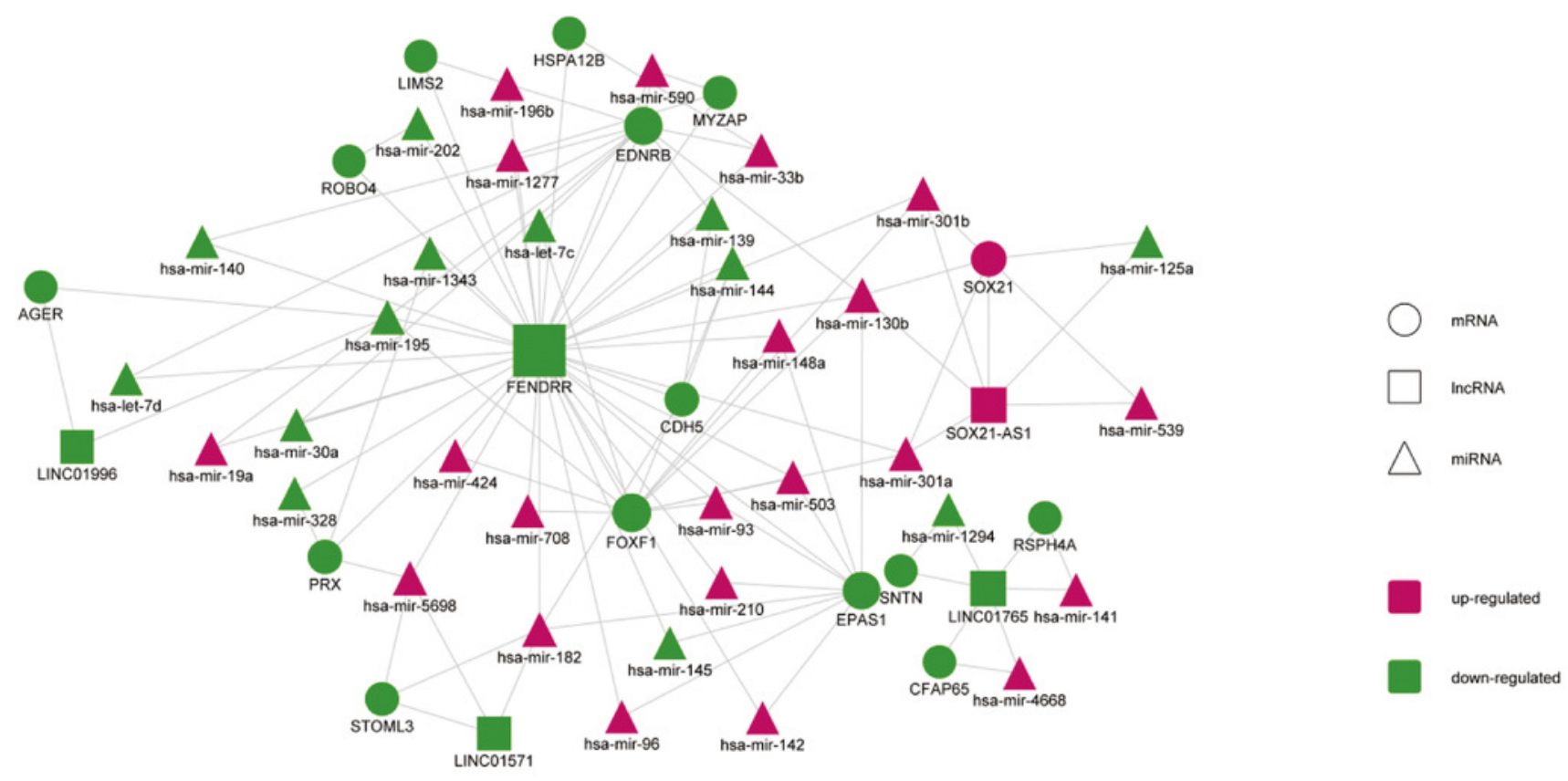


Figure 6

Kaplan-Meier survival curves for five ceRNA pairs associated with overall survival (A) EDNRB-FENDRR-hsa-mir-196b. (B) EPAS1-FENDRR-hsa-mir-148a. (C) FOXF1-FENDRR-hsamir-148a. (D) FOXF1-FENDRR-hsa-mir-195. (E) FOXF1-FENDRR-hsa-mir-301b.
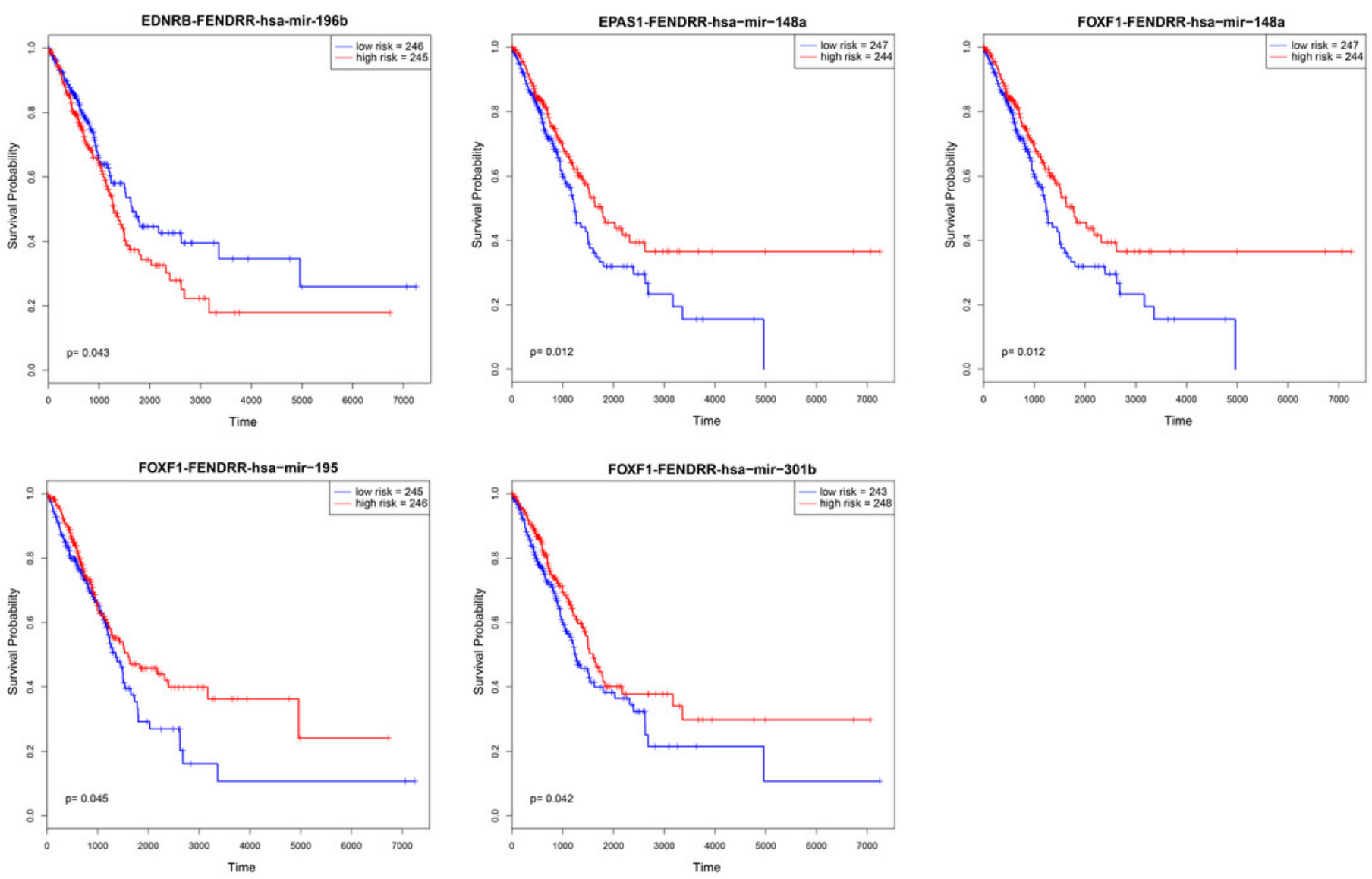
Figure 7

Co-expressed genes analysis for EPAS1

(A) EPAS1 co-expressed gene-set was enriched for motif "cisbp_M6212". (B) EPAS1regulated genes in LUAD

A

B
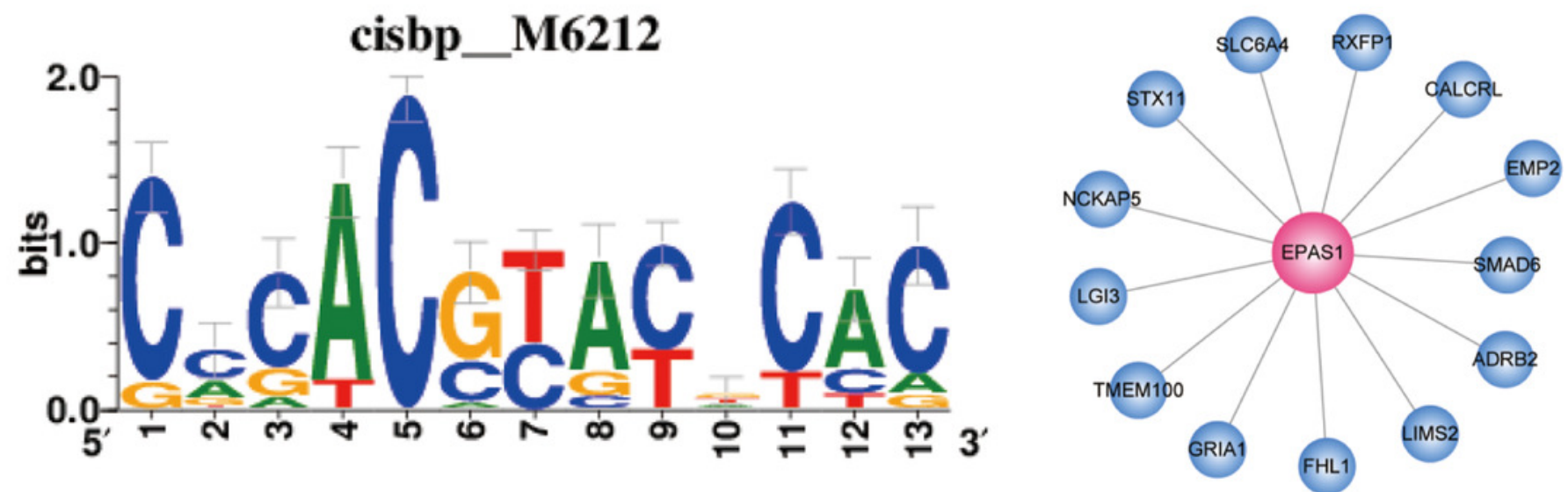
Figure 8

Validation of gene expression in GEO dataset

(A) EPAS1, FOXF1, and EDNRB expression level in GSE10072. (B) EPAS1, FOXF1 and EDNRB expression level in GSE32863. (C) FENDRR expression level in GSE85716 and GSE104854
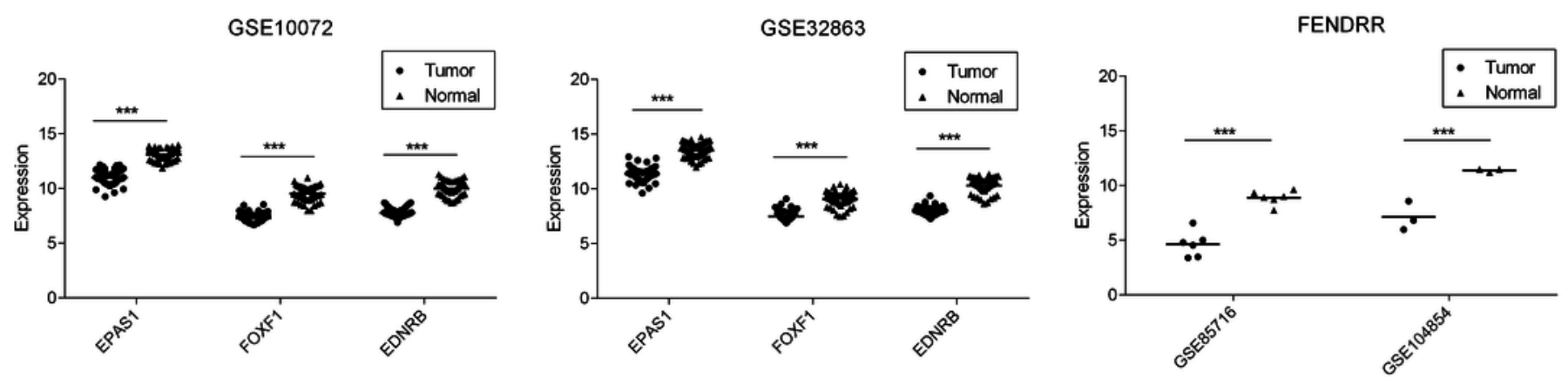


\section{Table $\mathbf{1}$ (on next page)}

Gene fold change in 5 ceRNA pairs. 
1 Table 1 Gene fold change in 5 ceRNA pairs.

\begin{tabular}{ccccccccc}
\hline & \multicolumn{2}{c}{ T1_vs_Normal } & \multicolumn{2}{c}{ T2_vs_Normal } & \multicolumn{2}{c}{ T3_vs_Normal } & \multicolumn{2}{c}{ T3_vs_Normal } \\
\hline Gene & log2FC & FDR & log2FC & FDR & log2FC & FDR & log2FC & FDR \\
\hline EPAS1 & -2.64 & $5.65 \mathrm{E}-97$ & -2.76 & $1.1 \mathrm{E}-127$ & -2.49 & $1.3 \mathrm{E}-64$ & -2.61 & $9.45 \mathrm{E}-41$ \\
FOXF1 & -2.44 & $6.68 \mathrm{E}-55$ & -2.96 & $2 \mathrm{E}-126$ & -2.58 & $1.2 \mathrm{E}-45$ & -2.63 & $8.73 \mathrm{E}-30$ \\
EDNRB & -3.27 & $2.09 \mathrm{E}-78$ & -3.75 & $9.2 \mathrm{E}-123$ & -3.38 & $3.4 \mathrm{E}-60$ & -2.94 & $8.47 \mathrm{E}-38$ \\
FENDRR & -3.48 & $2.85 \mathrm{E}-54$ & -4.37 & $2.7 \mathrm{E}-112$ & -3.78 & $1.62 \mathrm{E}-42$ & -3.89 & $1.94 \mathrm{E}-42$ \\
hsa-mir-148a & 1.51 & $1.31 \mathrm{E}-24$ & 1.46 & $1.51 \mathrm{E}-23$ & 1.45 & $4.7 \mathrm{E}-11$ & 1.51 & $1.07 \mathrm{E}-07$ \\
hsa-mir-195 & -1.98 & $6.47 \mathrm{E}-37$ & -2.25 & $5.03 \mathrm{E}-68$ & -2.28 & $2.9 \mathrm{E}-30$ & -2.08 & $6.23 \mathrm{E}-13$ \\
hsa-mir-196b & 3.00 & $1.59 \mathrm{E}-21$ & 4.42 & $7.94 \mathrm{E}-35$ & 2.98 & $5.6 \mathrm{E}-15$ & 4.21 & $1.08 \mathrm{E}-20$ \\
hsa-mir-301b & 3.48 & $2.62 \mathrm{E}-22$ & 4.14 & $7.95 \mathrm{E}-28$ & 2.94 & $9.5 \mathrm{E}-15$ & 3.02 & $1.82 \mathrm{E}-05$ \\
\hline
\end{tabular}

2 
Table 2 (on next page)

EPAS1, FOXF1, EDNRB and FENDRR expression in GEO dataset 
1

2 Table 2 EPAS1, FOXF1, EDNRB and FENDRR expression in GEO dataset

\begin{tabular}{ccccccccc}
\hline & \multicolumn{9}{c}{ mRNA } & \multicolumn{2}{c}{ lncRNA } \\
\cline { 2 - 9 } Study & \multicolumn{2}{c}{ EPAS1 } & \multicolumn{2}{c}{ FOXF1 } & \multicolumn{2}{c}{ EDNRB } & \multicolumn{2}{c}{ FENDRR } \\
\cline { 2 - 9 } & log2FC & FDR & $\log 2$ FC & FDR & $\log 2$ FC & FDR & $\log 2$ FC & FDR \\
\hline GSE10072 & -2.087 & $3.08 E-33$ & -2.228 & $2.17 E-35$ & -2.299 & $4.30 E-35$ & - & - \\
GSE32863 & -2.152 & $3.05 E-33$ & -1.616 & $2.69 E-28$ & -2.385 & $2.35 E-44$ & - & - \\
GSE85716 & - & - & - & - & - & - & -4.25009 & 0.000343 \\
GSE104854 & - & - & - & - & - & - & -4.12757 & $3.96 \mathrm{E}-08$ \\
\hline
\end{tabular}

3 